\title{
Uma Proposta de Embalagem Inteligente e Sustentável para a Indústria de Nobreaks
}

\author{
A Smart and Sustainable Packaging Proposal for the Nobreaks Industry \\ Una propuesta de embalaje inteligente y sostenible para la industria de Nobreaks
}

Recebido: 26/05/2021 | Revisado: 31/05/2021 | Aceito: 03/06/2021 | Publicado: 18/06/2021

\author{
Andreza Alexandre da Silva \\ ORCID: https://orcid.org/0000-0002-4050-5260 \\ Faculdade de Tecnologia Termomecanica, Brasil \\ E-mail: andreza.alesilva@gmail.com \\ Bianca Masumoto Costa \\ ORCID: https://orcid.org/0000-0002-7308-4431 \\ Faculdade de Tecnologia Termomecanica, Brasil \\ E-mail: biancamc@gmail.com \\ Breno Augusto Brito Ferraz \\ ORCID: https://orcid.org/0000-0002-2907-8587 \\ Faculdade de Tecnologia Termomecanica, Brasil \\ E-mail: ferraz-breno@ hotmail.com \\ Diogo Martins Gonçalves de Morais \\ ORCID: https://orcid.org/0000-0001-5681-4044 \\ Faculdade de Tecnologia Termomecanica, Brasil \\ E-mail: pro7113@cefsa.edu.br \\ Ebert Martins \\ ORCID: https://orcid.org/0000-0001-7054-4896 \\ Faculdade de Tecnologia Termomecanica, Brasil \\ E-mail: ebertmart@hotmail.com \\ Thaise Silva Martin \\ ORCID: https://orcid.org/0000-0003-4098-895X \\ Faculdade de Tecnologia Termomecanica, Brasil \\ E-mail: thaise.silva.da@gmail.com
}

\begin{abstract}
Resumo
O presente estudo tem o objetivo de apresentar um protótipo de embalagem, inteligente e sustentável, para o transporte de nobreaks de grande porte, em um estudo de caso real de uma indústria fabricante de nobreaks, localizada no município de Diadema (SP). Por meio do estudo de caso, o estudo concluiu que a construção e uso das embalagens são factíveis e viáveis, apresenta as características técnicas da embalagem e um estudo de viabilidade mercadológica e financeira, além de seus efeitos em todo o desenho da logística que envolve esses nobreaks. Além disso, o estudo confere à comunidade científica uma metodologia para inovação, com uma perspectiva para a sustentabilidade. Por fim, foi possível elaborar algumas ações ligadas a seis dos Objetivos de Desenvolvimento Sustentável - ODS, estabelecidas pelas Nações Unidas, que serão exploradas no decorrer do estudo.
\end{abstract}

Palavras-chave: Logística reversa; Embalagem inteligente; Material sustentável.

\begin{abstract}
The present study aims to present a smart and sustainable packaging prototype for the transport of large nobreaks, in a real case study of a manufacturer of nobreaks from Diadema (SP). Through the case study, the study concluded that the construction and use of packaging are feasible and feasible, presents the technical characteristics of the packaging and a study of market and financial feasibility, in addition to its effects on the entire logistics design that involves these nobreaks. In addition, the study gives the scientific community a methodology for innovation, with a perspective for sustainability. Finally, it was possible to elaborate some actions linked to six of the Sustainable Development Goals - SDGs, established by the United Nations, which will be explored in the course of the study.
\end{abstract}

Keywords: Reverse logistics; Smart packaging; Sustainable material.

\section{Resumen}

El presente estudio tiene como objetivo presentar un prototipo de envase inteligente y sostenible para el transporte de grandes nobreaks, en un caso de estudio real de un fabricante de nobreaks ubicado en el municipio de Diadema (SP). A través del caso de estudio, el estudio concluyó que la construcción y uso del empaque es factible y factible, presenta las características técnicas del empaque y un estudio de mercado y factibilidad financiera, además de sus efectos en todo el diseño logístico que involucra estos nobreaks. . Además, el estudio dota a la comunidad científica de una metodología de innovación, con perspectiva de sostenibilidad. Finalmente, fue posible elaborar algunas acciones 
vinculadas a seis de los Objetivos de Desarrollo Sostenible - ODS, establecidos por Naciones Unidas, que serán explorados en el transcurso del estudio.

Palabras clave: Logística inversa; Embalaje inteligente; Material sostenible.

\section{Introdução}

De acordo com dados fornecidos pelo Banco Mundial em 2018, o Brasil produziu cerca de 79 milhões de toneladas de lixo, com os plásticos representando 13,5\% desse volume, ou 11,3 milhões de toneladas, fazendo com que o Brasil seja o quarto maior produtor de resíduos plásticos do mundo (Kaza et al., 2018).

Em 2020, durante a pandemia, especula-se que esses números possam ter aumentado ainda mais, devido a mudanças e incorporações de novos hábitos, ou seja, neste período houve um aumento expressivo da compra de comidas prontas para consumo via delivery, comércio eletrônico e uso de materiais hospitalares descartáveis que foram os vilões para este aumento.

Se por um lado há o aumento expressivo do consumo de plástico pela população, por outro, é notável o crescente interesse da população pelas questões ambientais, o que faz emergir a necessidade de se estruturar novas formas de produção e distribuição que se caracterizem como sustentáveis (Shiozawa et al, 2020; Tomé, Morais \& Pereira, 2020).

Além desse conflito de interesse social, há também outra situação que aparentemente se contrapõe nos setores de produção, que é a emergente necessidade de se renovar os processos de produção com foco na sustentabilidade, diante do questionamento dos produtores sobre a viabilidade econômica e mercadológica desses novos modos de produção.

Diante disso, o presente artigo traz à margem estudos que apresentam casos de renovação de processo de produção com foco na sustentabilidade e apresenta, como objetivo geral, um estudo de caso próprio, em que se propõe um novo tipo de embalagem para o caso particular de uma indústria fabricante de nobreaks localizada no município de Diadema (SP), que tenha inteligência embarcada e seja sustentável, apresente agilidade no processo de preparação e montagem, seja rastreável e retornável.

Para a operacionalização do objetivo geral deste estudo, foram considerados os seguintes objetivos específicos:

a) Identificar o fornecedor de uma embalagem com as características apontadas, considerando os seus custos;

b) Elaborar propostas de soluções transformando embalagens passivas em embalagens inteligentes, ativas e conectadas, por meio de dispositivos autônomos;

c) Realizar o estudo da viabilidade financeira da embalagem.

A apresentação do estudo está dividida em seis partes: essa primeira introdutória, em que são expostos o contexto e objetivo do estudo; a seção 2, que apresenta os procedimentos metodológicos utilizados; a seção 3, que apresenta o referencial teórico; a apresentação e a análise dos resultados na seção 4; a seção 5, contendo as considerações finais, e por fim, a lista de referências utilizadas no artigo.

\section{Metodologia}

O presente estudo pode ser classificado como uma pesquisa exploratória, de natureza qualitativa, realizada por meio de um estudo de caso. Como afirma Pereira et al (2018) em geral, as pesquisas de estudo de caso são centradas em um fenômeno, o qual é descrito com a máxima profundidade possível, que no caso do presente estudo envolve o processo de embalagem e transporte de Nobreaks em uma indústria de Diadema, estado de São Paulo.

De acordo com Gil (2007), a pesquisa exploratória visa uma maior aproximação, uma maior familiaridade com o problema, explicitando-o. Na maioria das vezes, assume a condição de pesquisa bibliográfica ou de estudo de caso, segundo Gil (2007), e envolve levantamento bibliográfico, entrevistas e análises de elementos diversos, como o que se pretende desenvolver para uma indústria fabricante de nobreaks de grande porte, localizada no município de Diadema (SP). 
Desta forma, incialmente realizou-se revisão da literatura, pesquisas de mercado para a identificação de fornecedores e levantamento de custos e levantamento de dados da empresa utilizada neste estudo, tais como o volume e os custos atuais com o transporte de nobreaks de grande porte.

A partir dos dados levantados, foi possível comparar os custos envolvidos no processo e, então, avaliar a viabilidade financeira da embalagem proposta. A fim de facilitar a compreensão e desenvolvimento do projeto, o estudo foi dividido em sete etapas, a saber: Modelo de embalagem sustentável; Tecnologia e inovação a embalagem; Serviço de instalação e comissionamento de Nobreaks; Estudo de viabilidade econômica; Ciclo de vida da embalagem; Ações Sociais, e por fim, criação de valor ao negócio. A Figura 1 ilustra o percurso metodológico empreendido.

Figura 1 - Percurso metodológico.

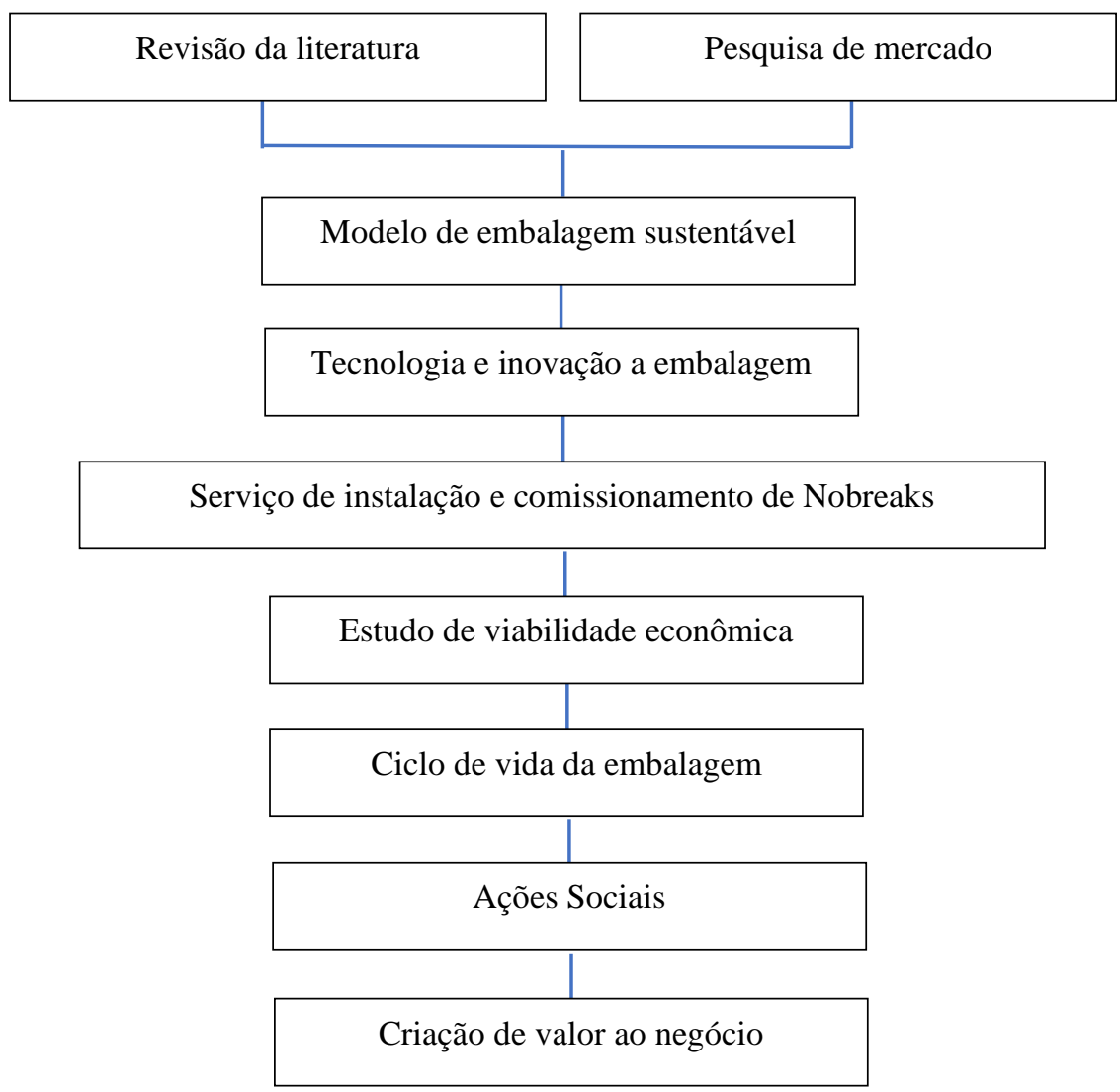

Fonte: Autores.

$\mathrm{Na}$ etapa intitulada de Modelo de embalagem sustentável realizou-se a identificação das embalagens existentes no mercado, a fim de escolher a mais adequada para o uso em nobreaks de forma a propor as alterações / melhorias para que o objetivo do desenvolvimento fosse alcançado. Buscou-se uma embalagem que garantisse a integridade do produto e o seu transporte, e atendesse também aos aspectos sustentáveis baseados nos três pilares da sustentabilidade (Econômico, Ambiental e Social).

Essa etapa foi concluída a partir da pesquisa de mercado e revisão da literatura para o reconhecimento de estudos semelhantes, prospeç̧ão de fornecedores a nível mundial, visitas e reuniões com fornecedores no estado de São Paulo - SP, e estudo de fichas técnicas dos possíveis produtos disponíveis pelos fornecedores.

Na etapa denominada Tecnologia e inovação a embalagem realizou-se o estudo de tecnologias a ser agregadas a embalagem com o objetivo de criar valor ao produto, como sensores de vibração, controle de temperatura e rastreamento em 
tempo real, tornando a embalagem inovadora.

Da mesma maneira, essa etapa do estudo contou com os resultados da revisão da literatura e pesquisa de mercado, para o reconhecimento das tecnologias comerciais existentes que atendesse a demanda requerida.

$\mathrm{Na}$ etapa denominada Serviço de instalação e comissionamento de Nobreaks, foi realizado uma reconfiguração do fluxo de trabalho do serviço de instalação e comissionamento de Nobreaks, redesenhado de modo a otimizar os custos envolvidos no processo. Esse estudo foi feito sobre o processo padrão adotado hoje pela empresa envolvida.

$\mathrm{Na}$ etapa denominada Estudo de viabilidade econômica, foi realizado um levantamento de custos da situação atual para confrontar com novos valores e calculando TIR e PAYBACK de dois cenários: cenário atual (com a demanda atual da empresa estudada) e com cenário pessimista (com redução de 30\% da demanda de Nobreaks na região delimitada de atendimento).

Na etapa denominada Ciclo de vida da embalagem, realizou-se um estudo do tempo de vida das embalagens propostas versus o tempo de vida das embalagens atuais e buscou-se mensurar a redução de materiais utilizados. O estudo foi feito com estimativa do tempo de vida útil da embalagem proposta, e análise crítica do descarte dos materiais após o uso.

Desta maneira, partiu-se para a etapa denominada Ações Sociais, onde se elaborou uma proposta de desenvolvimento de um programa de extensão para capacitação de membros da comunidade no entorno da empresa, além de destinar recursos e ações para atualizações e treinamento internos. Análise feita em cima da demanda técnica e dados da empresa de estudo.

Por fim, na etapa denominada Criação de valor ao negócio, buscou-se destacar o valor agregado da proposta, em forma de fluxo de serviço, que foi o reflexo da otimização de recursos de embalagens, com a substituição de alternativa reutilizável e reciclável, bem como a garantia de um fluxo com maior segurança e rapidez na instalação, com otimização de recursos financeiros e ambientais, que pode ser sentido pelo cliente.

\section{Resultados e Discussão}

Com a finalidade de tornar a apresentação dos resultados mais rica, considerando que o percurso metodológico empregado também se mostrou eficiente para a proposta de novos estudos, em outros estudos de caso, optou-se por apresentar os resultados de maneira capitular, associando cada resultado à etapa descrita no percurso metodológico desse estudo.

\subsection{Resultados da revisão da literatura científica e mercado}

Para atender ao objetivo geral desse estudo, que é a proposta de um novo tipo de embalagem inteligente e sustentável para a indústria de nobreaks, buscou-se identificar, na literatura científica, estudos que sustentam a ideia de viabilidade dos produtos ou processos sustentáveis.

Para a compreensão do leitor sobre o conceito de desenvolvimento sustentável, considerou-se neste artigo a ideia de Triple Bottom Line, cunhado por Elkington (1998), mesmo que o mesmo autor tenha sugerido uma expansão dessa ideia em 2018 (Elkington, 2018), a abordagem de desenvolvimento sustentável na esfera ambiental, econômica e social é uma base conceitual suficiente para os fins desse estudo.

Em resposta a esses conflitos de interesse, constata-se que práticas sustentáveis podem trazer um retorno financeiro para o produtor, e certamente, um retorno positivo para todo o sistema produtivo e meio ambiente, como mostrou o estudo de Oliveira Neto et al. (2015), que avaliou a vantagem ambiental e a econômica da implantação da produção mais limpa de um fabricante de plásticos usando reciclagem em um ciclo fechado de polímeros.

Os resultados indicaram vantagens ambientais com redução da poluição em 3.507.470,8 kg e vantagens econômicas de $\mathrm{R} \$ 75.384,00$ mensais pelo reaproveitamento de $21.600,0 \mathrm{~kg}$ de resíduos de polímeros com a implantação da produção mais limpa (Oliveira Neto et al, 2015). 
Os estudos de Sousa et al (2016) consideram a logística reversa como um diferencial competitivo, proporcionando responsabilidade socioambiental e redução de custos dos insumos de produção, trazendo retorno financeiro e competitividade entre outras empresas.

Segundo Domingues, Valentim e Miranda (2020), por meio de um estudo de caso em uma indústria de cosméticos do interior de Minas Gerais, a preservação do lucro da instituição está diretamente relacionada aos investimentos em sustentabilidade apresentados pela empresa.

Outros estudos, como àqueles realizados por Hammes et al. (2020), mapearam a logística reversa de uma empresa de embalagens industriais no norte de Santa Catarina, e após a análise destes custos, comprovou-se que a logística reversa pode gerar uma receita positiva para a empresa, além de originar ganhos ambientais e sociais.

Existem também diversos estudos que apresentam técnicas e métodos sustentáveis, que reduzem o custo operacional em diversos seguimentos, como apresentado por Roque e Pierri (2019), Soares et al. (2020) e Santos et al. (2020), que mostraram o efeito que técnicas denominadas "verdes" tem sobre a redução de custos dos empreendimentos.

Por fim, registra-se nesse referencial teórico que há estudos que também mostram que a tecnologia embarcada em processos logísticos pode trazer benefícios, como a capacidade de se gerenciar o ciclo de pedido, remover atividades que não agregam valor, agilizar entregas e, por conseguinte, atender às metas de desempenhos logísticos, como os estudos de Merlotto, Moori, e Lopes (2021), Areias et al. (2020), Cordeiro et al. (2018), Morais, Frey e Savassa (2016), e por fim, Bendlin et al. (2014).

Todos os estudos apontados seguem em linha com as constatações de Donaire (1994) que assevera que a consideração ambiental nos meio de produção não corresponde apenas à um custo para as organizações, mas, também, pode gerar diversos benefícios, sendo: redução de custos em decorrência do menor consumo e do uso adequado dos recursos; geração de lucro mediante ao reaproveitamento de resíduos; economia de recursos; descoberta de novas matérias-primas e processos de produção; fortalecimento e melhoria da imagem da empresa e crescimento das vendas, devido ao desenvolvimento de produtos ambientalmente favoráveis.

Além da revisão da literatura, pesquisou-se quais seriam os fabricantes de embalagens semelhantes àquelas que serão propostas para este estudo de caso. O Quadro 1 apresenta os dados desse levantamento.

Quadro 1 - Fabricantes de embalagens para a indústria brasileira.

\begin{tabular}{|l|l|c|c|c|c|c|}
\hline \multicolumn{1}{|c|}{ Empresa } & \multicolumn{1}{|c|}{ Ramos atendidos } & Retornável & Inteligente & Reciclável & Upcycling & Local \\
\hline Reciclapack & $\begin{array}{l}\text { Automotivo / Transporte / } \\
\text { Fabricante de motores e } \\
\text { geradores / Transportadora / } \\
\text { Metalurgia / }\end{array}$ & $\mathrm{X}$ & $\mathrm{X}$ & $\mathrm{X}$ & $\begin{array}{c}\text { São } \\
\text { Paulo } \\
\text { SP }\end{array}$ \\
\hline Rackmetal & Automotivo & $\mathrm{X}$ & & & & $\begin{array}{c}\text { São } \\
\text { Paulo } \\
\text { SP }\end{array}$ \\
\hline Alveotech & $\begin{array}{l}\text { Automotivo / Eletroeletrônico } \\
\text { / Alimentício / Farmacêutico }\end{array}$ & $\mathrm{X}$ & & $\mathrm{X}$ & & $\begin{array}{c}\text { Valinhos } \\
\text { SP }\end{array}$ \\
\hline $\begin{array}{l}\text { Ativva } \\
\text { Soluções }\end{array}$ & Automotivo & $\mathrm{X}$ & & & & $\begin{array}{c}\text { Itatiba } \\
\text { SP }\end{array}$ \\
\hline Nefab & Automotivo & $\mathrm{X}$ & & & & Portugal \\
\hline
\end{tabular}

Fonte: Autores. 
Observa-se que nenhuma das fabricantes pesquisadas possui embalagens que sejam consideradas inteligentes e sustentáveis, se considerados os critérios de serem retornáveis, recicláveis e upcycling, além de terem algum tipo de inteligência embarcada.

Diante desta situação, optou-se por identificar potenciais fornecedores de embalagem produzidas com material reciclável, além de fornecedores de dispositivos para a inteligência embarcada (sistema de rastreamento; sensores de temperatura, de umidade e para acompanhar as condições de transporte), obtendo, assim, a embalagem com as características almejadas.

\subsection{Resultados do modelo de embalagem sustentável}

Dentro as opções estudadas, a caixa do fornecedor "Ativva Soluções” foi a que mais atendeu a necessidade do projeto, por ser uma caixa com suas laterais intercambiáveis e ajustadas a medida necessária. O produto é feito com PEBD (Polietileno de Baixa Densidade) e possui uma base única capaz acomodar Nobreaks de médio e grande porte. A Figura 2 apresenta o modelo.

Figura 2 - Modelo de embalagens prospectada para o projeto da empresa Ativva Solutions.

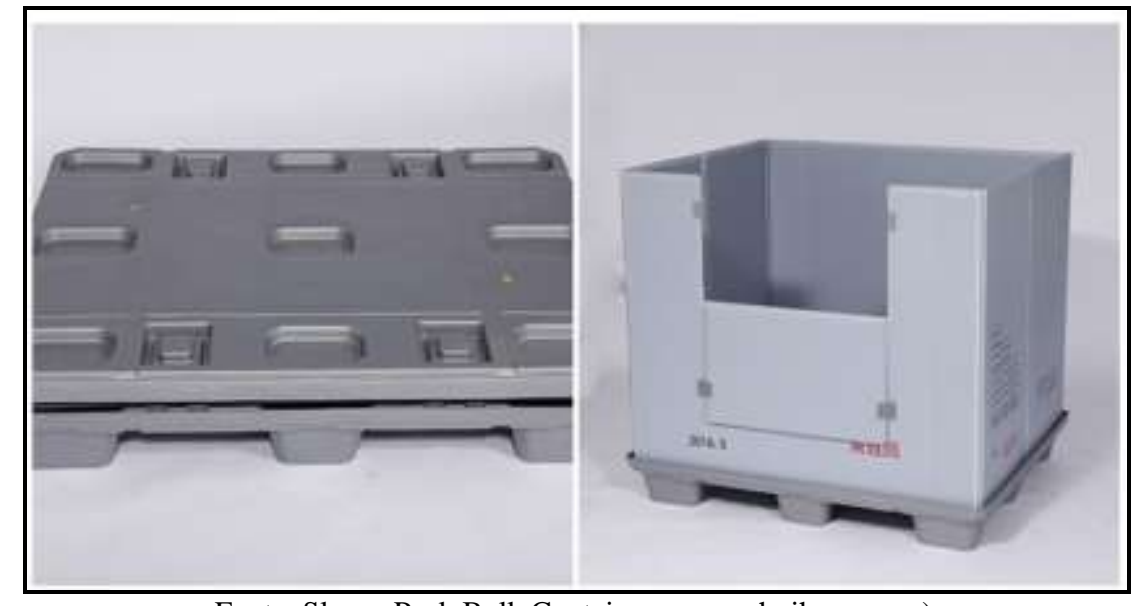

Fonte: Sleeve Pack Bulk Container. www.choihope.com).

A embalagem é considerada reutilizável, retornável, leve, desmontável, que proporciona melhor ergonomia e otimização do transporte, já que também é empilhável. Além das atribuições já citadas ela é muito versátil, pois pode ser produzida de vários tamanhos e ser aplicada ao abrigo e transporte de produtos para diversos segmentos.

\subsection{Resultados da tecnologia e inovação da embalagem}

O sistema de rastreamento será composto por um microcontrolador e um shield, placa de circuito conectada ao Arduino, capaz de realizar a conexão GPRS. Seguindo uma arquitetura como mostrada na Figura 3 a seguir. Os sensores de temperatura, umidade e impacto transmitirão informações para o microcontrolador, este irá filtrar dados relevantes e, através do shield SIM808, enviará ao cliente uma mensagem de texto contendo apenas o conteúdo indispensável. 
Figura 3 - Arquitetura do sistema de rastreamento.

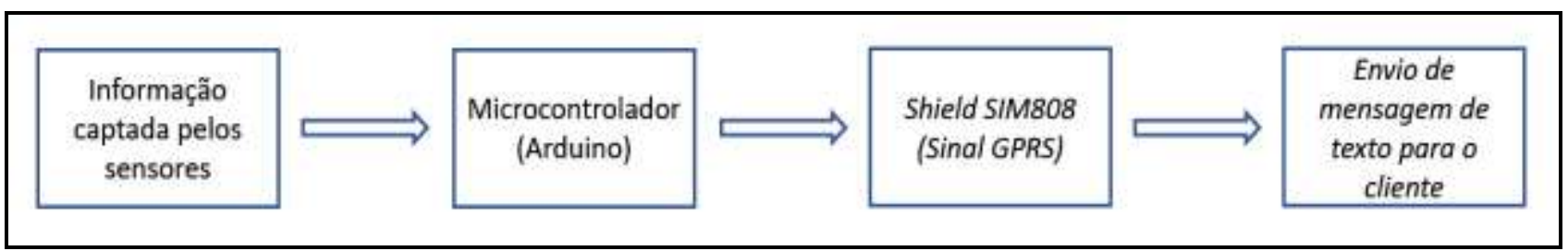

Fonte: Autores.

O controlador proposto para este projeto é o Arduino UNO que por sua vez uma plataforma open source ou hardware para prototipagem eletrônica, projetada com um microcontrolador Atmel AVR com suporte para entrada/saída dados já embutido, com linguagem de programação padrão baseado no em $\mathrm{C} / \mathrm{C}++$.

Este por sua vez será integrado com o shield SIM808 a rede de dados móveis. Este módulo é capaz de realizar conexões a internet via rede de telefonia e gerar seus dados de localização através do GPS, Sistema de Posicionamento Global.

Além deste módulo, serão utilizados sensores, como sensores de umidade e temperatura, para acompanhamento das condições de armazenamento e sensores do tipo giroscópio e acelerômetros para verificar e acompanhar as condições de transporte tais como quedas e posição da caixa, sensores magnéticos qual Funcionam através de um contato elétrico e um ímã.

A função do ímã é manter o sensor acionado e, no caso de abertura de porta ou janela, há afastamento dos contatos, enviando imediatamente um sinal para o microcontrolador para se gerar um alarme. Um display qual servem como uma forma de exibir uma informação para o usuário, de forma que ela possa ser facilmente vista e compreendida e um teclado matricial quais são geralmente utilizados em aplicações na qual o usuário precisar interagir com um sistema, como computadores, calculadoras, controles remotos entre outros (Figura 4).

Figura 4 - Sistemas de hardware do módulo de controle.

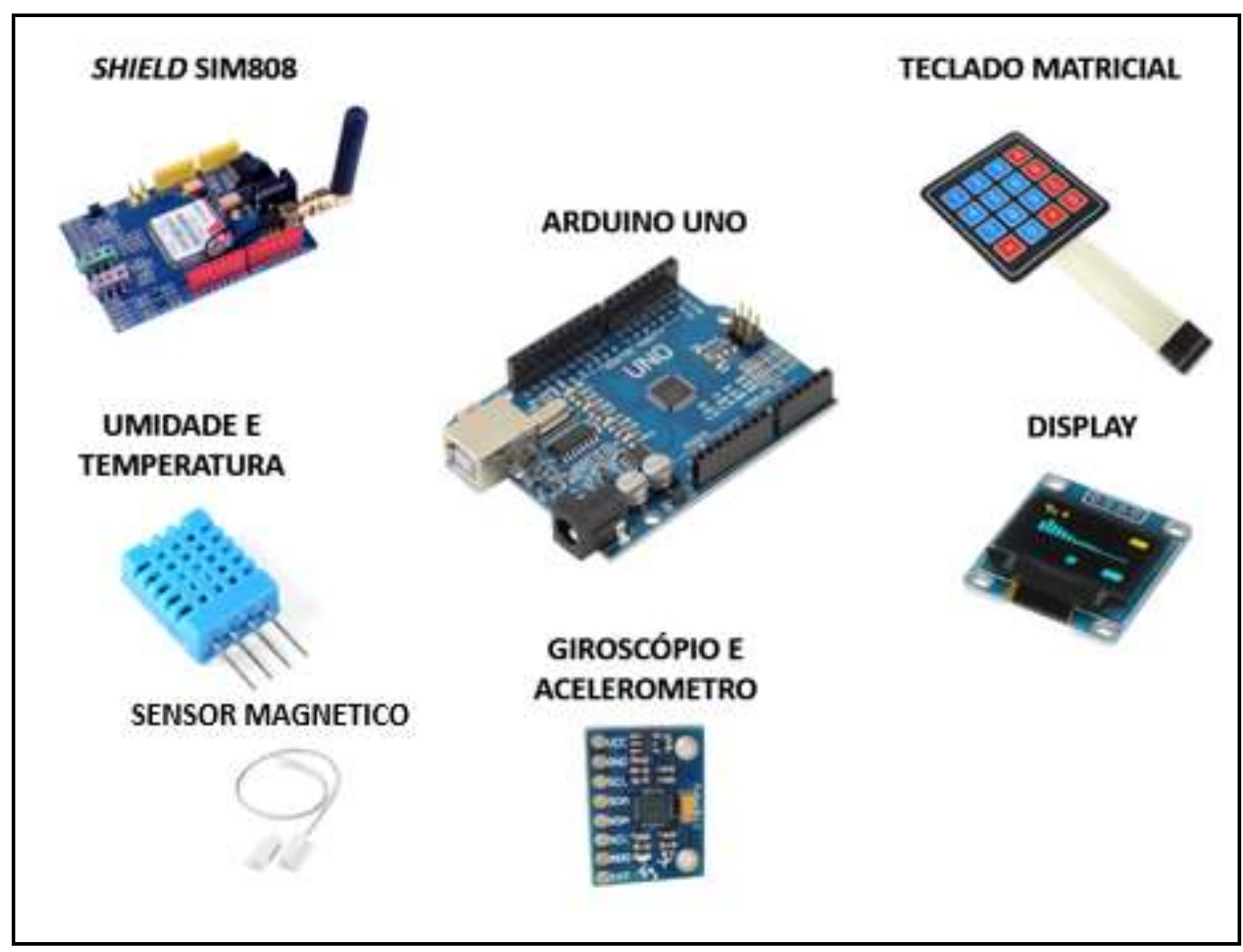

Fonte: Autores. 
O sistema completo, como mostrado na Figura 5, deverá ser programado de tal forma a permitir que os dados relevantes de localização, condição de armazenamento e transporte sejam enviados ao cliente por meio de mensagens de texto, facilitando assim o controle de todo o comportamento do produto ao longo da viagem.

Figura 5 - Protótipo do sistema completo e integrado a caixa para transporte.

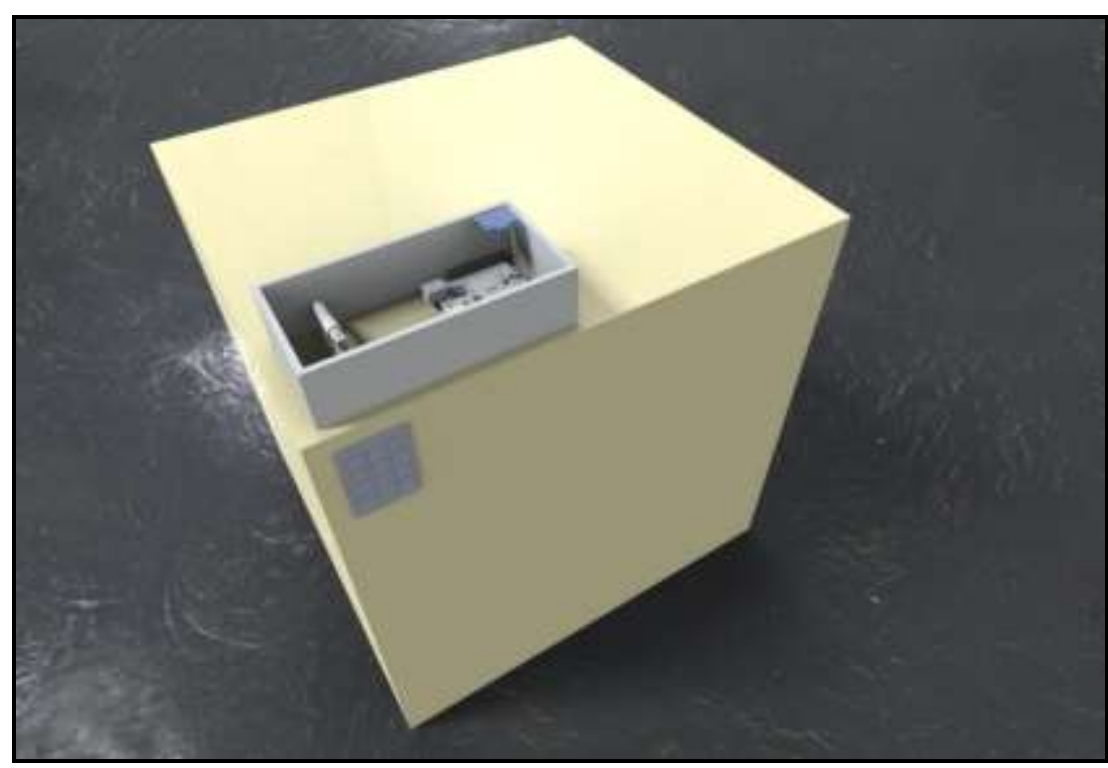

Fonte: Autores.

Uma vez concluído o protótipo do sistema completo, realizou-se o estudo da instalação e comissionamento dos Nobreaks que serão transportados.

\subsection{Resultados da instalação e comissionamento de nobreaks}

Ao analisar o serviço da instalação e comissionamento da indústria de Nobreaks, foi identificado uma otimização de fluxo de trabalho.

No processo atual, a empresa se dispõe de duas equipes: Uma para frete e outra para instalação e comissionamento. O frete, em sua grande maioria, é feito por terceiros com empresas contratada e seguro. Após a chegada do produto, o cliente liga e solicita a instalação, no qual a segunda equipe se destina ao cliente e realiza o serviço de instalação e comissionamento. Essa segunda equipe utiliza de recursos da empresa como veículos e ferramental, além dos gastos com transporte, hospedagem e alimentação. Na Figura 6 está o esboço de ambos os processos. 
Figura 6 - Fluxo do processo atual e do processo proposto.

\section{Processo Atual}

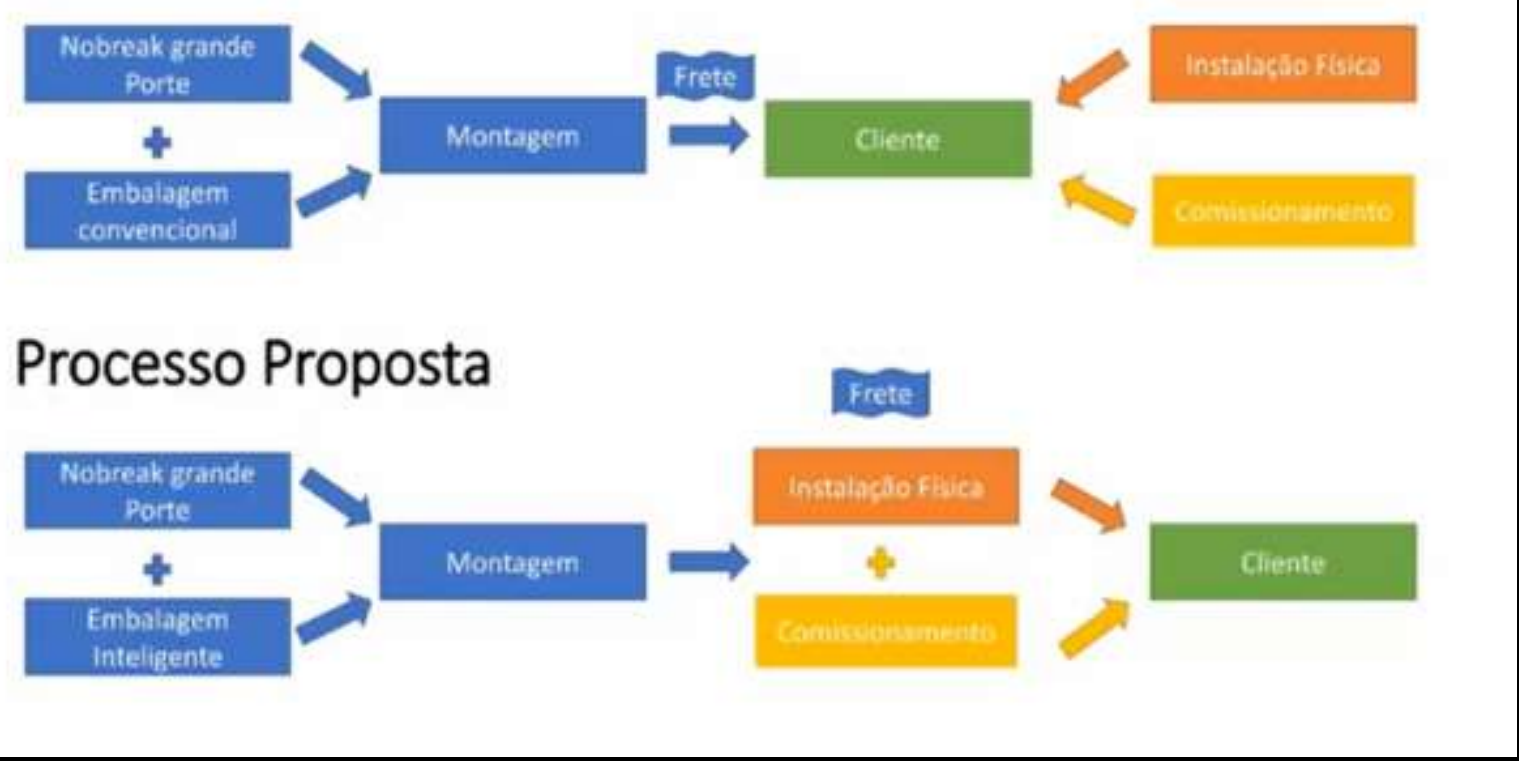

Fonte: Autores.

A proposta foi agregar a uma única equipe, de forma interdisciplinar a função de transporte, instalação e comissionamento do equipamento, otimizando recursos e promovendo a maior conhecimento e qualificação da equipe.

\subsection{Resultados do estudo de viabilidade econômica}

Como escopo do projeto o novo fluxo e de embalagem foi aplicado ao estado de São Paulo-SP. Pelo histórico de venda da empresa, essa região se destina $12,30 \%$ das vendas de grande porte da empresa, essas vendas correspondem à 180 vendas por ano, 15 vendas por mês, $80 \%$ dessas vendas geram a entregam de um único equipamento, que leva ao número de 12 fretes mensais.

Alocando esses 12 fretes em duas equipes físicas (6 fretes/mês), teríamos um tempo estimado de viagem e retorno das caixas à empresa de 1 mês. Em cima dessa demanda original, e considerando $20 \%$ a mais de caixas, foi proposto a compra de 18 caixas.

Para custeio, foi considerado o investimento das caixas, kit de sensores, adaptação ferramental do veículo e diárias e custos da equipe.

Com esses dados, foram gerados dois estudos financeiros com fluxo de caixa, um com o cenário atual de demanda, e outro com cenário pessimista, considerando menos $30 \%$ da demanda de venda de frete. O Fluxo de caixa está apresentado na Figura 7. 
Figura 7 - Fluxo de caixa dos modelos de serviço/produto atual versus modelo de serviço/produto proposto.

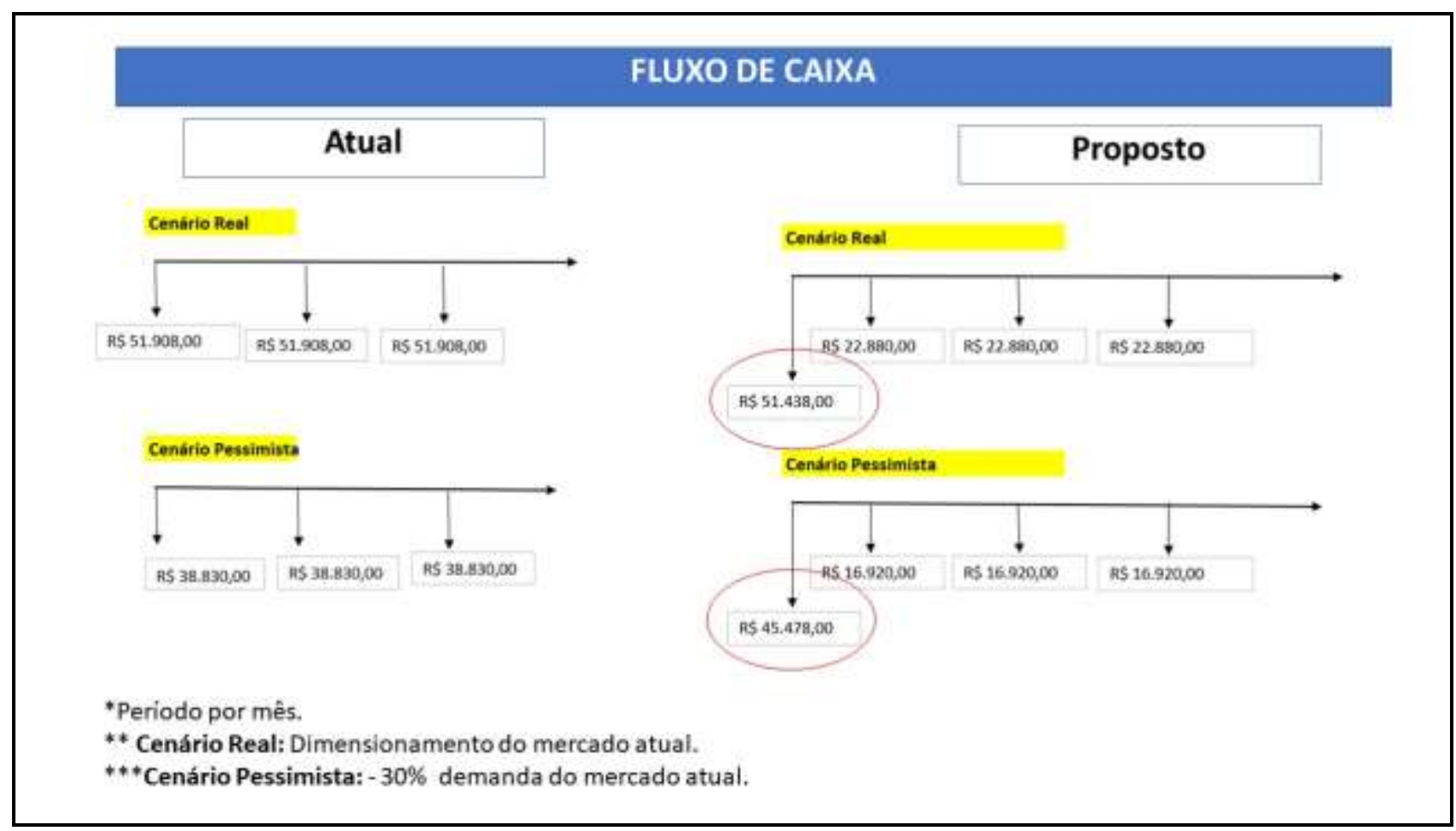

Fonte: Autores.

O ganho financeiro no cenário atual é imediato, e no cenário pessimista a TIR equivaleria a 325\% e o payback simples de dois meses.

\subsection{Resultados do Ciclo de vida da embalagem}

Destinou-se após o a vida útil da caixa, que o PEBD será destinado a cooperativas de reciclagem e os sensores será destinada a empresa especializado em tratamento e destinação de resíduos eletrônicos. A Figura 8 ilustra a comparação do processo atual com o processo proposto.

Figura 8 - Comparação dos materiais descartados no processo atual versus processo proposto.

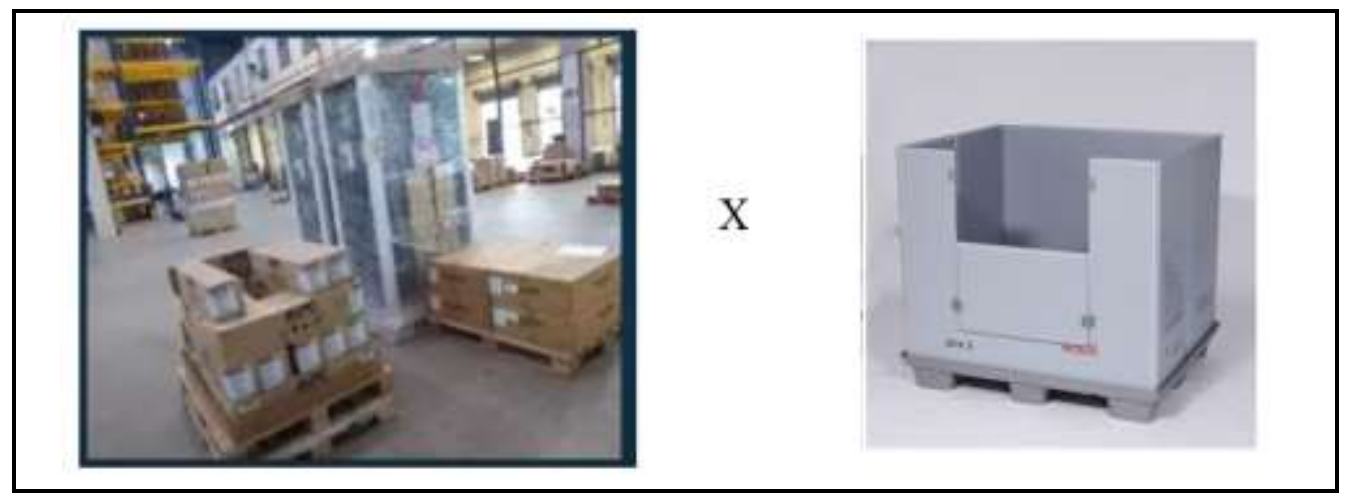

Fonte: Autores.

A embalagem desenvolvida possui uma vida útil de 5 anos (60 viagens), ou seja, 1 caixa proposta pode apresentar uma redução de $600 \mathrm{~kg}$ de material de embalagem de uso único, considerando a quantidade inicial de caixas estimadas, essa redução chega 11,8 toneladas de material de embalagem ao longo da vida útil. 


\subsection{Resultados das ações sociais}

No âmbito social o projeto estipulou um programa de capacitação interna de da comunidade entono à empresa de manutenção das caixas, gerando conhecimento e atendendo a demanda interna. Essa capacitação será gerada pelo saving gerado no próprio projeto. Os impactos sociais e até mesmo ambientais do projeto estão alinhados a seis Objetivos de Desenvolvimento Sustentável (ODS), da ONU, ilustrados na Figura 9.

Figura 9 - Objetivos de Desenvolvimento Sustentável.

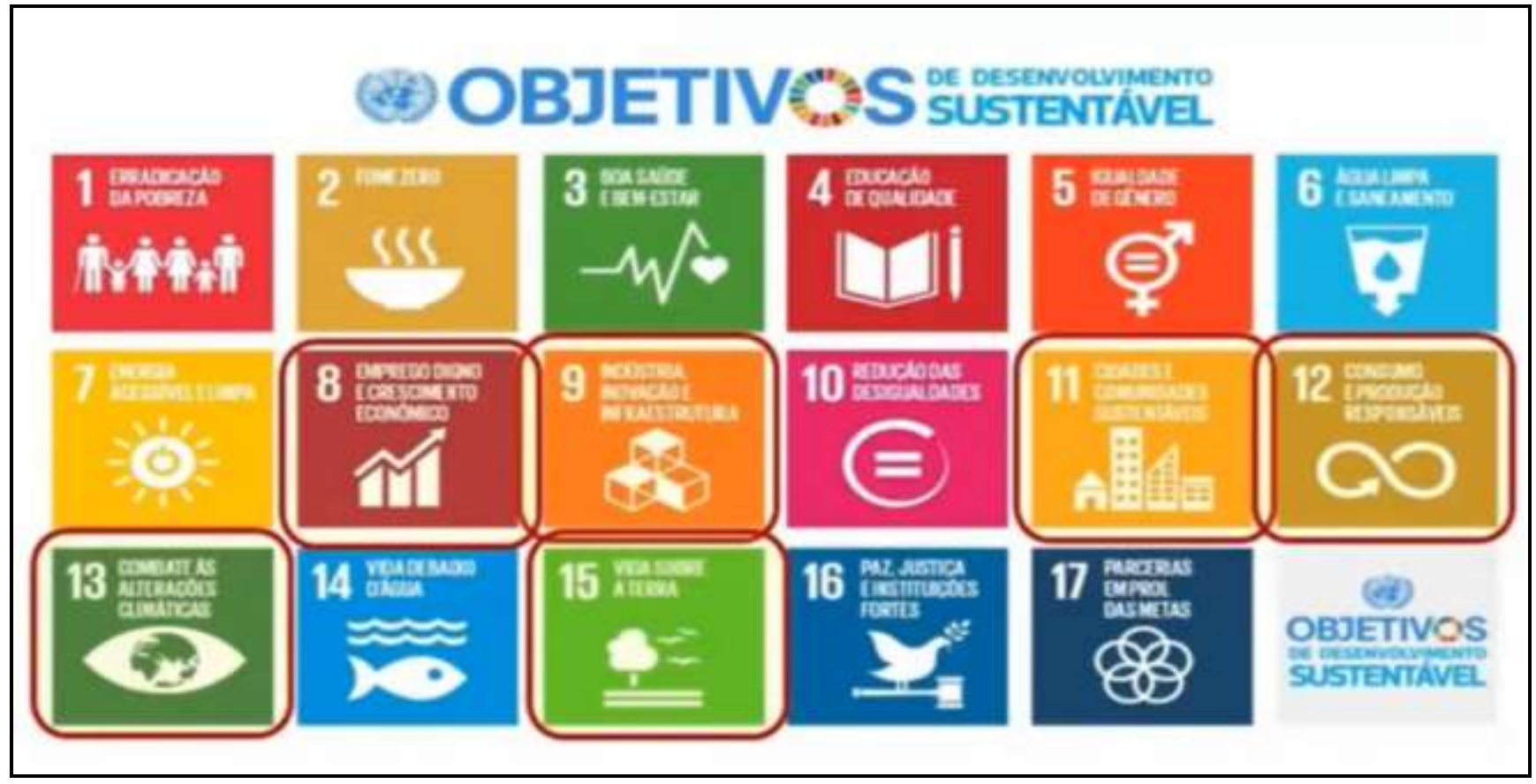

Fonte: ODS. https://odsbrasil.gov.br.

Os seis Objetivos de Desenvolvimento Sustentável (ODS), da ONU, relacionados ao projeto, são: (ODS 8) Emprego Digno e Crescimento Econômico: quando estimula o emprego e crescimento do time envolvido no processo; (ODS 9) Industria Inovação e Infraestrutura: quando cria valor com uso de sensores na embalagem; (ODS 11) Cidades e Comunidades Sustentáveis: quando expande treinamento a comunidade e expande a relações sociais da empresa; (ODS 12) Consumo e Produção Responsável: quando passa utilizar embalagem reutilizável e deixa de usar materiais com curtos períodos de vida útil; (ODS13) Combate a alterações climáticas: quando reduz o uso de combustíveis fósseis e poluentes; (ODS 15) Vida Sobre a Terra: quando reduzimos o consumo de madeira.

\subsection{Resultados da criação de valor ao negócio}

Na Rich Picture apresentada na Figura 10 (a) e (b), o fluxo do produto até chegar ao cliente é alterado e agregado tecnologia de rastreabilidade e integridade, no qual gera segurança sensível a consumidor como valor. 
Figura 10 - Rich Picture da geração de valor (a) Processo atual; (b) Processo proposto.

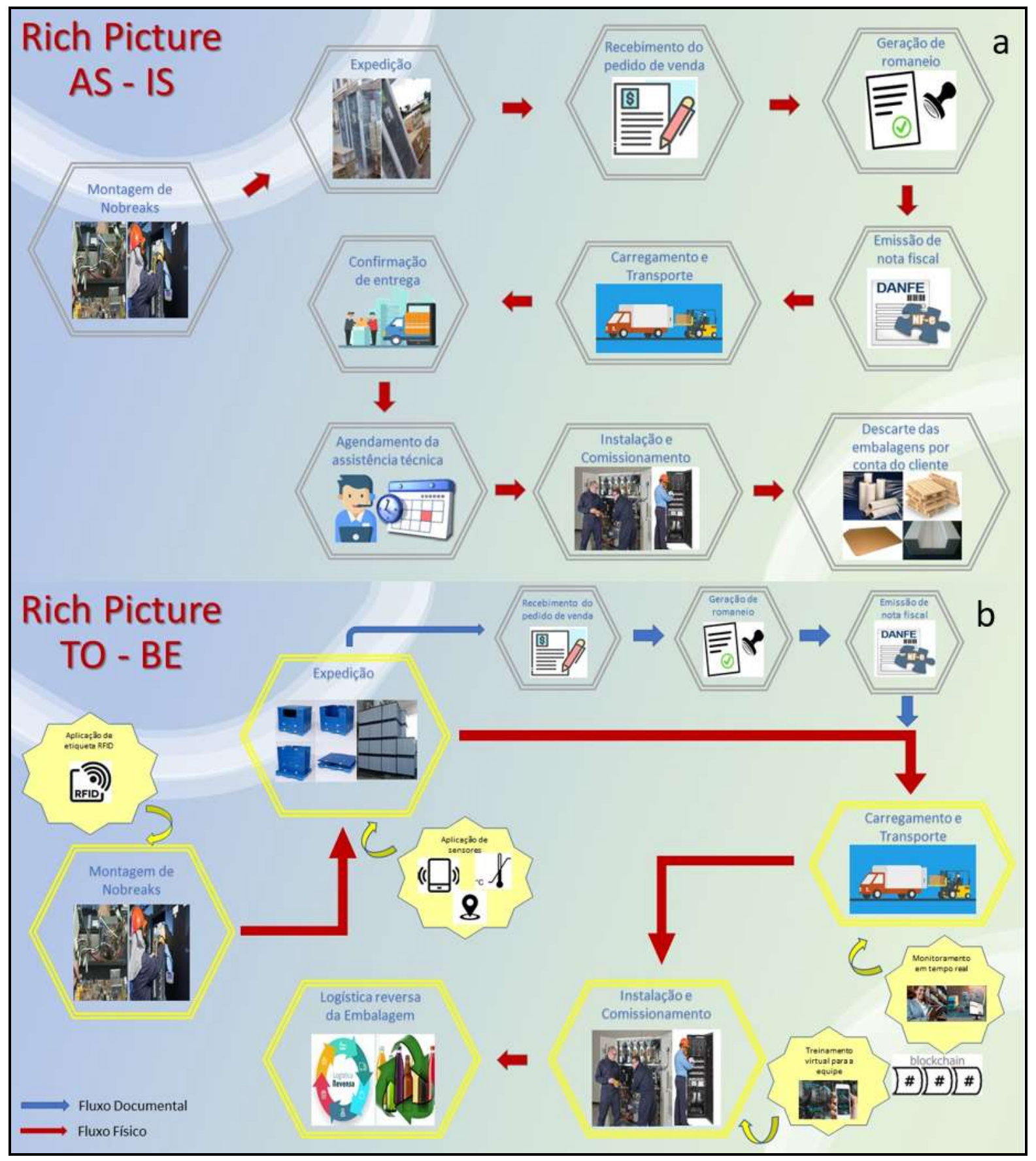

Fonte: Autores.

Outro aspecto sensível ao consumidor é o tempo de instalação e disponibilidade de uso do equipamento, que no fluxo proposto, possui etapas otimizadas, sensível ao consumidor.

\section{Conclusão}

O presente estudo mostrou, por meio do protótipo apresentado, com suas características, que é possível desenvolver uma embalagem que transporte com segurança o material envolvido no estudo de caso, e que atende aos requisitos de sustentabilidade social e ambiental.

Ao embarcar tecnologia de rastreamento e alterar o fluxo do serviço de instalação e comissionamento, agregou-se 
valor ao produto, melhorando os índices de segurança e atendimento ao cliente.

A viabilidade financeira nesse modelo também foi provada pelos custos mensurados e modelo de fluxo proposto. Essa análise corrobora a discussão trazida por Sousa et al (2016), Roque \& Pierri (2019), Hammes et al. (2020), além de Domingues, Valentim E Miranda (2020) sobre o retorno financeiro que o investimento em sustentabilidade pode trazer aos negócios como um todo.

Toda a racionalização de processo proposto faz convergência com os interesses das ODS e cria valor à sociedade, conforme assevera Donaire (1994) sobre os diversos benefícios para a organização que considera o desenvolvimento sustentável em seus processos.

Além da viabilidade de construção e uso da embalagem inteligente, enquanto alternativa sustentável para o transporte de nobreaks da indústria estudada, o presente estudo confere, enquanto metodologia, uma proposta para a inovação e sustentabilidade.

É evidente que, por se tratar de um estudo de caso, a pesquisa traz limitações de escopo, como a abrangência de atendimento, limitada aos municípios paulistas citados.

Desta maneira, como oportunidades para estudo futuros, recomenda-se novos estudos com ampliado escopo de atendimento para outras regiões, produtos e empresas.

\section{Referências}

Areias, A. A., Cruz Júnior, J. C., Faria, L. C. de, \& Yamaji, F. M. (2020). A logistical and economical approach to coordinating a biomass supply chain, including energy characteristics. Research, Society and Development, 9(8), e757986050.

Bendlin, L., Senff, C. O., Pedro, J. J., \& Stafin, O. O. S. (2014). Custos de produção, expectativas de retorno e riscos associados ao plantio de eucalipto na região do Planalto Norte - Catarinense/Brasil. In: Congresso Brasileiro de Custos. 21, Natal, Brasil. <https://anaiscbc.emnuvens.com.br/anais/article/view/3677>.

Cordeiro, S. A., Silva, M. L. da, Oliveira Neto, S. N., \& Oliveira, T. M. (2018). Simulação da Variação do Espaçamento na Viabilidade Econômica de um Sistema Agroflorestal. Floresta e Ambiente, 25 (1).

Domingues, H., Valentim, T. J., \& Miranda, G. M. do C. (2020). Responsabilidade socioambiental: o caso de uma indústria de cosméticos do interior de Minas Gerais, Brasil. Research, Society and Development, 9(7), e359973056.

Donaire, D. (1994). Considerações sobre a influência da variável ambiental na empresa. Revista de Administração de Empresas (RAE), 34 (2).

Elkington, J. (1998). Partnerships from cannibals with forks: The triple bottom line of 21st-century business. Environmental Quality Management, 8(1), 37-51.

Elkington, J. (2018). 25 years ago I coined the phrase "triple bottom line." Here's why it's time to rethink it. Harvard Business Review. < https://hbr.org/2018/06/25-years-ago-i-coined-the-phrase-triple-bottom-line-heres-why-im-giving-up-on-it>.

Gil, Antonio Carlos (2007). Como elaborar projetos de pesquisa. Atlas.

Hammes, G., Nilson, M., Silva, F. L. da, Rodriguez, C. M. T. \& Lezana, A. G. R. (2020) Mapeamento dos custos de logística reversa em uma indústria de embalagens. Exacta, 18 (3), p. 668-685.

Kaza, Silpa; Yao, Lisa C; Bhada-Tata, Perinaz \& Van Woerden, Frank. (2018). What a Waste 2.0: A Global Snapshot of Solid Waste Management to 2050. Urban Development. World Bank. https://openknowledge.worldbank.org/handle/10986/30317.

Morais, D.M.G., Frey, Julia Hille \& Savassa, Lucas Pires. (2016). Estudo da distribuição ótima das unidades coletoras de materiais reciclados em São Bernardo do Campo - um mapa para o investidor social. Revista Eletrônica Gestão e Serviços, 7 (2), p. 1715-1726.

Merlotto, W. F., Moori, R. G., \& Lopes, Y. (2021). Tecnologia da Informação como um Alavancador de Desempenho em Prestadores de Serviços Logísticos. Revista Alcance, 28(1), 52-66.

Oliveira Neto, Geraldo Cardoso de; Shibao, Fábio Ytoshi; Filho, Moacir Godinho \& Chaves, Luiz Eduardo Carvalho. (2015) Produção mais Limpa: Estudo da Vantagem Ambiental e Econômica na Reciclagem de Polímeros. Interciência, 40 (6).

Objetivos de Desenvolvimento Sustentável (2020). Indicadores Brasileiros para os Objetivos de Desenvolvimento Sustentável. https://odsbrasil.gov.br.

Pereira A. S. et al. (2018). Metodologia da pesquisa científica. UFSM.

Roque, R. A. L., \& Pierri, A. C. (2019). Uso inteligente de recursos naturais e sustentabilidade na construção civil. Research, Society and Development, 8(2), e3482703. 
Research, Society and Development, v. 10, n. 7, e19610716547, 2021

(CC BY 4.0) | ISSN 2525-3409 | DOI: http://dx.doi.org/10.33448/rsd-v10i7.16547

Santos, C. A. A. S. dos, Lucena, M. S. de, Moraes, W. dos S., Silva, L. C., Silva, D. E. C., Serra, M. A. A. de O., \& Façanha Filho, P. de F. (2020). Material compósito de argamassa e polímero: uma opção sustentável para a construção civil e reutilização de pneus inservíveis na cidade de Açailândia, Brasil. Research, Society and Development, 9(7), e538974591.

Shiozawa, Simone, Junger, Alex Paubel, Morais, Diogo Martins Gonçalves de, Oliveira, Eduardo Cézar de, Oliveira, Victor Inácio de \& Borges, Luciana Guimarães Naves Lemos. (2020). A educação ambiental no contexto dos bacharelados em Engenharias e Administração das Universidades do ABC. Revista de Casos e Consultoria, 11(1), e1115.

Soares, J. A. B., Gomes, L. F., Cunha, F. N., Tavares, G. G., Giongo, P. R., Silva dos Santos, L. N., Teixeira, M. B., Lopes Sobrinho, O. P. \& Paixão, C. F. C. da. (2020). Uso de resíduo da indústria sucroalcooleira para a produção de mudas de quiabo. Research, Society and Development, 9(6), e62963229.

Souza, D., Oliveira, G. de, Mendonça, H., \& Cruz, P. (2016). Logística Reversa e Sustentabilidade. Revista Inovação, Projetos e Tecnologias - IPTEC, 4(1).

Tomé, I. M., Morais, D. M. G., Pereira, R. S. (2020) A educação ambiental nos cursos superiores de Tecnologia da Área de Gestão e Negócios e nos Bacharelados em Administração. Revista Meio Ambiente e Sustentabilidade, 9(19). 